\title{
Modeling the Effects of Different Environmental Stimuli on Radio Wave Propagation
}

\author{
Aaron Don M. Africa, Ara Jyllian A. Abello, Joaquin Miguel B. Lalusin \\ Department of Electronics and Communications Engineering \\ De La Salle University, Manila \\ 2401 Taft Ave., Malate, Manila 1004, \\ Philippines, aaron.africa@dlsu.edu.ph
}

\begin{abstract}
Antennas are devices used either as a transmitter wherein electric current is supplied to the antenna then transmits that current as a radio wave or a receiver wherein radio waves are intercepted and converted to electric current. These electromagnetic radio waves are used for long distance communications. When designing antennas for the use of propagating radio waves, it is important to take into account that there can be possible losses or attenuations in real world applications. So in order for an optimal deployment of an antenna, modeling under harsh conditions should be good practice. This paper will consider the possible environmental conditions under which antennas typically operate at such as rain, fog, and other atmospheric gases. The modeling will be done by using the Phased Array System Toolbox that is available in MATLAB. The group will then make a script that will plot the various losses due to different environmental stimuli.
\end{abstract}

Key words: radio wave propagation, environmental stimuli, wireless communication, monostatic radar, MATLAB.

\section{INTRODUCTION}

A reliable and consistent communication between antennas is critical when implementing wireless communications systems. Because of this, it is important to understand how the environment around the antennas can interact with the radio signals it is transmitting. There are many factors that can affect the propagation of radio waves such as ionospheric irregularities [1], the curvature of the Earth, free space, refraction, reflection [2], and many more. Currently there is an increasing demand for bandwidth [3], as the strain on a network increases due to the load it is being put through the performance of a communications system will suffer because the signals it is propagating will likely be attenuated or be subjected to noise. A solution to this problem is the inevitable utilization of bandwidth in the higher frequency bands [4]. However, this solution is not a one size fits all type of proposition. This is because several propagation systems work only at a specific frequency band. Therefore, predictive models for propagations losses can play a significant role in the design of a communications system, they can help identify key specifications to consider when implementing an antenna such as frequency reuse and transmission power. This can help in mitigating the performance impact on the antenna when it is operating.

With that said, the aim of this study is to understand how the weather can impact the performance of radio wave propagation by modelling a monostatic radar antenna using MATLAB. This is to provide an insight as to how much performance is lost due to rain, fog, and other atmospheric gases. After looking at the attenuations caused by the different weather conditions, thinking of ways to help compensate for the losses should be easier. Designing antennas with the foresight to include losses is incredibly important as this will affect how they will perform in the real world.

\section{BACKGROUND OF THE STUDY}

Radio waves travel from a transmitter to a receiver over a distance through a medium which can affect radio propagation (RF propagation) [5]. Different factors which affect RF propagation are not limited to the medium and the frequency but the different things or objects which may be on the path of the radio wave [6,7]. Due to these, radio waves may reflect, refract, and diffract. Therefore, when designing a radio communication system, it is important to consider these factors $[8,9,10]$.

Different types of RF propagation also influence the radio wave. One type is the free space propagation wherein radio waves are travelling in an empty space wherein there are no objects that affect the signal and the atmosphere or weather conditions are not present [11]. Another is the ground wave propagation wherein the radio waves are travelling along the surface of the ground and is ideal for RF over a short distance propagation using the low frequency or medium frequency part of the radio frequency spectrum [12,13]. Next is the ionospheric propagation wherein radio waves are propagated in the ionosphere or the atmosphere of the earth and utilizes the high frequency portion of the radio frequency spectrum $[14,15,16]$. Lastly, tropospheric propagation wherein radio waves are propagated through the troposphere which is 
Aaron Don M. Africa et al., International Journal of Emerging Trends in Engineering Research, 8(9), September 2020, 5307 - 5313

located above the earth's surface and uses the very high frequency portion of the radio frequency spectrum $[17,18]$.

All these types are subject to signal path loss since signals are attenuated when travelling. Main factors which determine the path loss is the transmitter power and antenna parameters such as the location, height, polarization, and gain. This path loss may be calculated and predicted to determine what affects the propagation of signal and find a more efficient way of propagation to reduce the attenuation [,19,20]. Some environmental factors such as rain and fog are unavoidable. Therefore, analysis of the entire wireless communication is essential to achieve less attenuation [21]. Understanding of the system and how path loss affects it will give way to improvement of the wireless communication system.

\section{STATEMENT OF THE PROBLEM}

Radio waves do not travel in an empty space. Simply put there are many factors that can potentially affect the performance of signal propagation. Since they are electromagnetic waves, they are affected by phenomena such as reflection, refraction, absorption, diffraction, scattering, and polarization. In light of this, it is important to note how different weather conditions can affect the radio waves travelling through them. Especially since the frequency band of these radio waves are being filled up with more and more users, it is getting harder for systems to propagate radio waves. One other solution to combat the increasing congestion bandwidth problem is to move over to the higher and underutilized frequencies such as the millimeter-wave frequency bands (mmWave, $30-300 \mathrm{GHz}$ ). However, this poses major challenges as well since higher frequency signals propagate poorer than lower frequency ones. Therefore, it is paramount to optimize the performance of communications systems wherever possible.

\section{SIGNIFICANCE OF THE STUDY}

Due to the increasing usage of the electromagnetic spectrum signal propagation has also become increasingly difficult. Because of this, the researchers wanted to help find a way to properly optimize radio wave propagation and have decided to take a look at where else in a wireless communications system can there be possible attenuations. When deploying antennas for wireless communication, it is important to consider the impact of the current environmental conditions. This is especially true when constructing antennas in areas where there are harsh weather conditions. It is also important to note that signals do not travel in a vacuum, therefore it is necessary to consider what parameters or specifications of an antenna to modify in order for it to overcome the possible attenuations that it will definitely face once it is operating. Planning ahead of time is of utmost importance when designing antennas or other wireless communication systems, furthermore this habit should be ingrained especially to students and young engineers who are just starting out in the industry. In addition to that, this study aims to provide an insight into how much performance is lost but also when the attenuations occur, i.e. at what distance, at how much rainfall, or how severe the weather might be.

\section{DESCRIPTION OF THE SYSTEM}

In this study, a radar system will be used to model the possible attenuations that might affect it. The radar range equation used to calculate the received signal power of a monostatic radar shall be used.

\section{METHODOLOGY}

\subsection{Rain}

A.Initialize variables such as frequency, range, angle of elevation and polarization

B. Initialize values for different rates of rain.

C. Very Light Rain: $0.25 \mathrm{~mm} / \mathrm{h}$

D. Light Rain: $1 \mathrm{~mm} / \mathrm{h}$

E. Moderate Rain: $5 \mathrm{~mm} / \mathrm{h}$

F. Heavy Rain: $20 \mathrm{~mm} / \mathrm{h}$

G. Extreme Rain: $60 \mathrm{~mm} / \mathrm{h}$

H. Calculate the signal loss due to rain for each rain rate using the rainpl function

I. Plot the rain attenuation with respect to the frequency

\subsection{Fog}

A.Initialize variables such as frequency, range, temperature

B.Initialize values of water density for different fog thickness

C. Medium Fog: $0.05 \mathrm{~g} / \mathrm{m}^{\wedge} 3$

D. Thick Fog: $0.5 \mathrm{~g} / \mathrm{m}^{\wedge} 3$

E.e. Calculate the signal loss due to fog for each water density using the fogpl function

F.f. Plot the fog attenuation with respect to the frequency

\subsection{Atmospheric Gas}

A.Initialize variables such as frequency, range, temperature

B.Initialize values of dry air pressure at $101300 \mathrm{~Pa}$

C.Calculate the signal loss due to atmospheric gas using the gaspl function

D.Plot the fog attenuation with respect to the frequency

\subsection{All Effects on a $110 \mathrm{GHz}$ radar}

A.Include all the variables needed for the previous weather conditions.

B.Set the rain rate to heavy rain at $20 \mathrm{~mm} / \mathrm{h}$; fog to thick fog at $0.5 \mathrm{~g} / \mathrm{m}^{\wedge} 3$

C.Plot all the losses with respect to the frequency

\subsection{Total Loss of a $110 \mathrm{GHz}$ radar}

A.Calculate the total loss given the previous parameters of the $110 \mathrm{GHz}$ radar using the phased array toolbox. 
B.Check if the total loss calculated is correct by adding the previously calculated loss for each of the losses.

\section{REVIEW OF RELATED LITERATURE}

There have been several studies that have tackled radio wave propagation. One such study modeled the radio wave propagation in an underground mine [22]. In the paper, they provided an overview of the characteristics of the wireless communication system. The authors described the mine environment and the extraction of minerals as complex, dangerous, hazardous, and aggressive. Due to the minerals and solid walls found in an underground mine, the propagation of radio waves suffers. The study found that communication performed the best at the $2.4 \mathrm{GHz}$ band when compared to 5.8 and $60 \mathrm{GHz}$ respectively but was unsuccessful at identifying a proper solution for mitigating the performance lost. Another study looked at how centimeter and millimeter-waves are attenuated in urban areas [23]. Since 5G is an emerging technology which is supposed to operate at the higher frequency bands which are more commonly called as centimeter and millimeter waves, they are subjected to symptoms which electromagnetic waves in the higher end of the spectrum suffer from. Most notably, the challenge of proper signal propagation in areas with a large amount of obstructions [24,25]. This is because these signals get absorbed by solid objects such as buildings, cars, trees, and even people. In the study about $5 \mathrm{G}$ propagation, the authors provided correction models so that the path loss may be mitigated by fitting the proper path loss model to the system.

As discussed earlier, RF propagation experiences path losses since it travels in a medium wherein some objects or interferences are present. One study presented and analyzed the path loss data and the corresponding information used for modeling radio wave propagation in a smart campus environment [26]. Here, calculations and measurements on three different routes are done to gather information on path loss data from the base station transmitter to the mobile receiver. The relationship of the collected information and the network parameters were understood through correlation analysis. In another paper, path loss of mobile radio propagation was predicted with the use of Artificial Neural Networks (ANN) [27]. The paper introduced the use of ANNs for predicting the path loss since it could obtain a prediction with enough accuracy. It was then compared to traditional path loss prediction methods and was proven to be effective in calculating the total path loss of the transmitted radio waves.

There are different factors that affect radio wave propagation. In one study, the effects of gaseous absorption and the attenuation due to clouds were modeled [28]. Here, a $72 \mathrm{GHz}$ terrestrial link having weathers sensors was used and the attenuation model was compared and examined with current data from a W/V-band Terrestrial Link Experiment. Another paper studied the effect of the location of an antenna and its polarization on radio wave propagation [29]. The method used in modeling the radio wave propagation in this study is called the ray-tracing method. Both the vertical polarization and the horizontal polarization of the antenna were simulated, and the median field intensity was applied for radio wave propagation evaluation. The results of the simulations indicate that the position of an antenna affects the radio wave coverage in the tunnel. In another study, the dyadic Green's function was used in predicting the electric field value for a deterministic RF propagation model [30]. Climate conditions were considered in the model that was proposed through changing constants in the medium the radio wave is travelling. The results of the model were differentiated and were proven to be satisfactory. Five signal attenuation prediction models due rain have been evaluated for earth-space links in one study [31]. Since RF propagation for satellite communications are located at frequencies above $10 \mathrm{GHz}$, it is affected by weather conditions, especially rain. The researchers of this study utilized different prediction models for rain attenuation and determined which of the models is optimal for satellite communication. Results showed that three of the five models studied were performing well in that region. In another study, a model for radio wave attenuation caused by rain has been proposed [32]. Multiple works on how rain affects radio wave attenuation by other researchers have been compared and discussed in the paper. After careful analysis, the researchers have proposed a new prediction model for radio wave attenuation caused by rain.

\section{THEORETICAL CONSIDERATIONS}

Designing and modeling wireless communications systems provide a basis to designers and engineers as to how the system will perform. Since the performance of an antenna can vary depending on its specifications, it is important to know which parameters are viable in each situation. Compensating for the attenuations that may affect the signals that are being propagated is essential. In this study, the authors looked at different weather conditions and varied their intensity in order to compare the losses across the different situations.

\section{DATA AND RESULTS}

Figures 1 to 9 shows the simulations of the propagation.

\subsection{Propagation Loss Due to Rain}

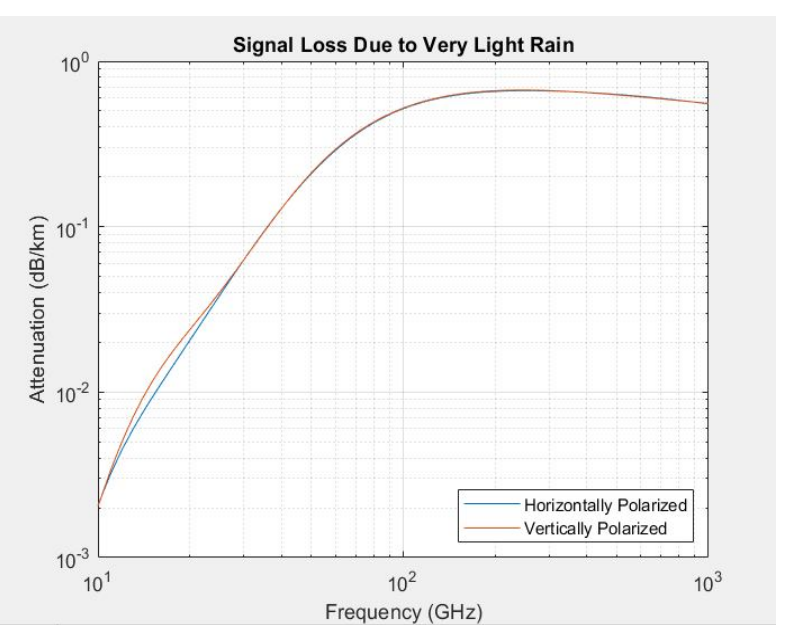

Figure 1: Signal Loss Due to Very Light Rain 
Aaron Don M. Africa et al., International Journal of Emerging Trends in Engineering Research, 8(9), September 2020, 5307 - 5313

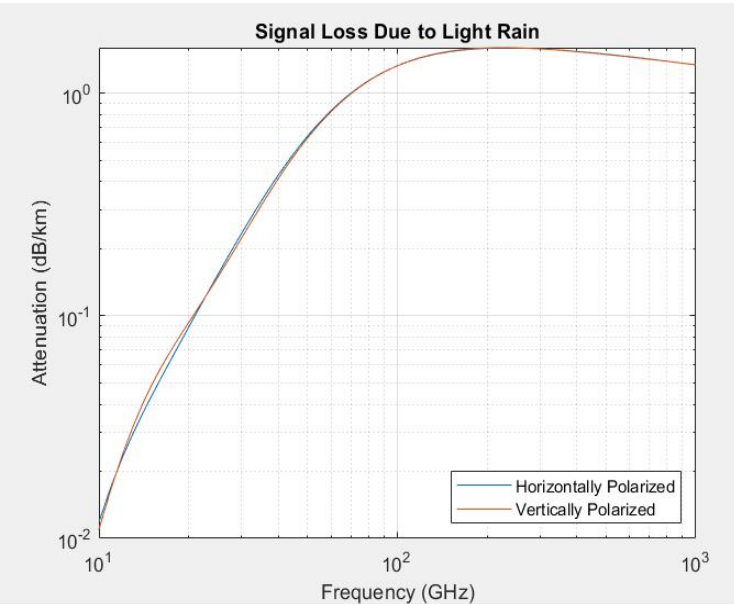

Figure 2: Signal Loss Due to Light Rain

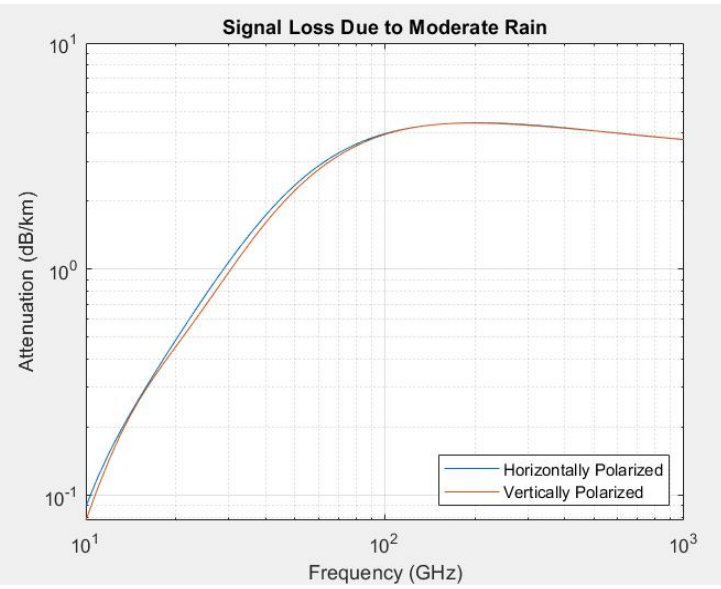

Figure 3: Signal Loss Due to Moderate Rain

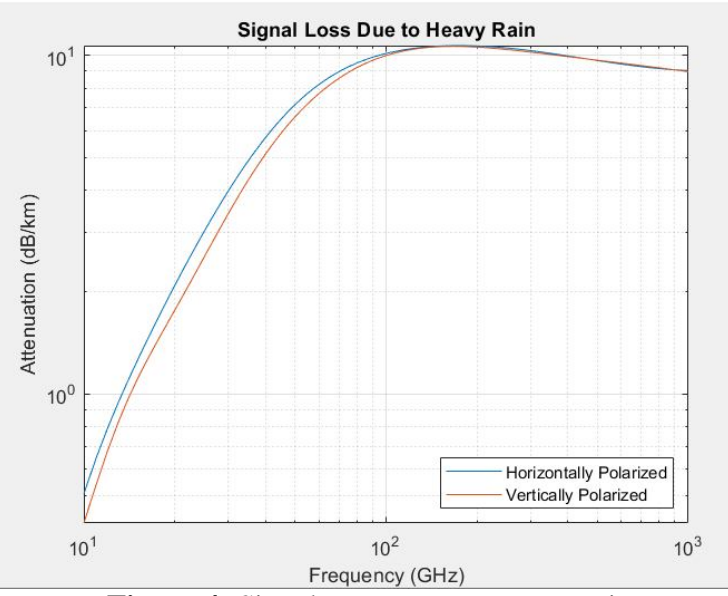

Figure 4: Signal Loss Due to Heavy Rain

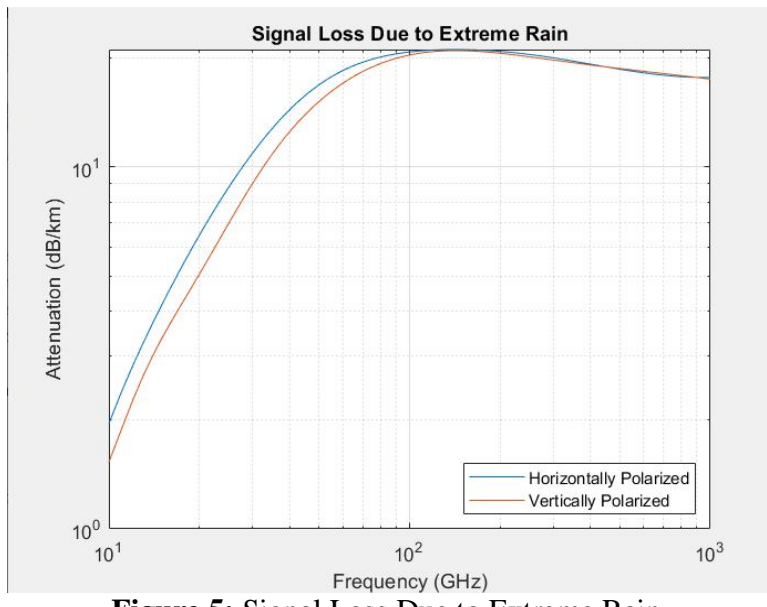

Figure 5: Signal Loss Due to Extreme Rain

9.2. Fog

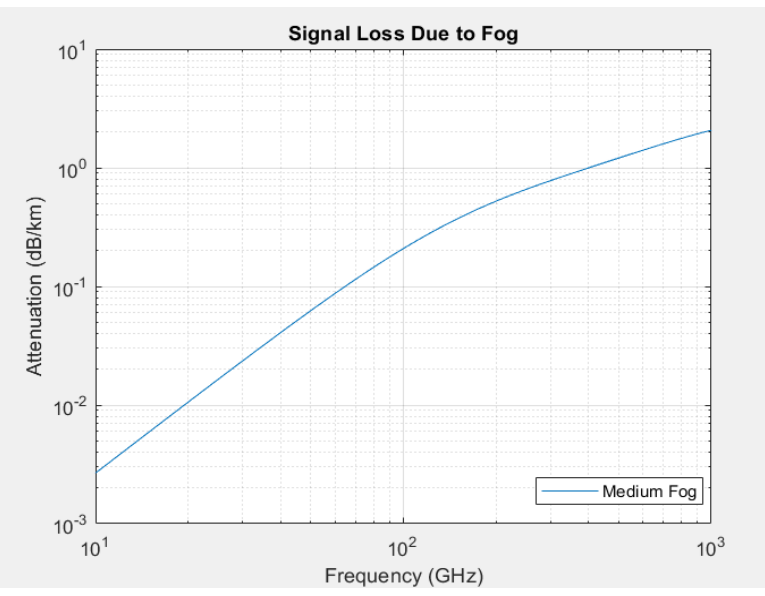

Figure 6: Signal Loss Due to Medium Fog

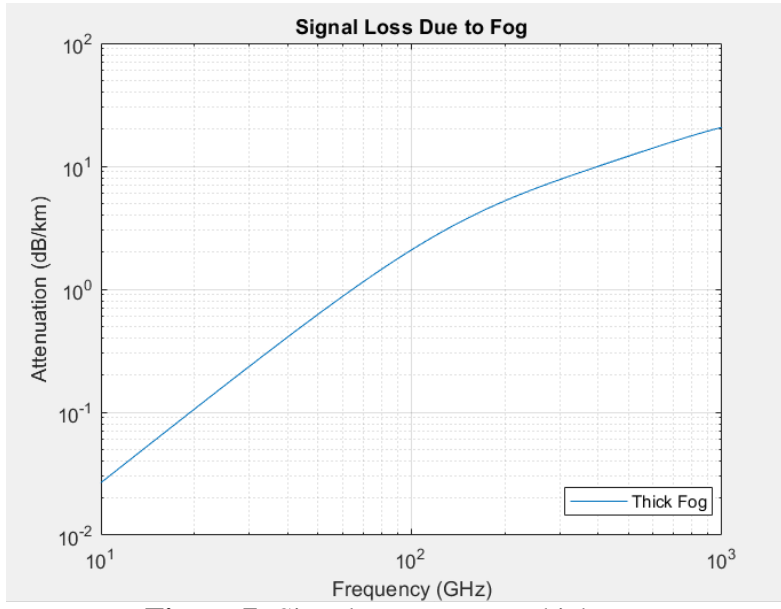

Figure 7: Signal Loss Due to Thick Fog 


\subsection{Atmospheric Gases}

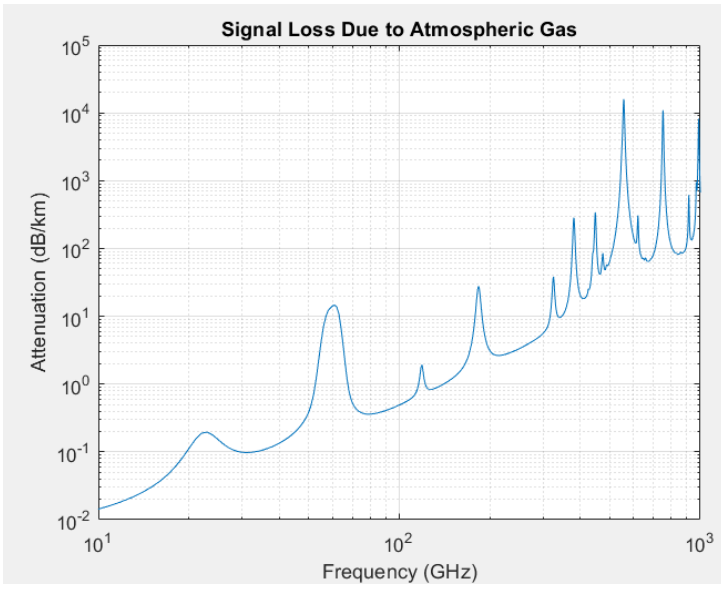

Figure 8: Signal Loss Due to Atmospheric Gas

\subsection{Signal Loss of a $110 \mathrm{GHz}$ Radar}

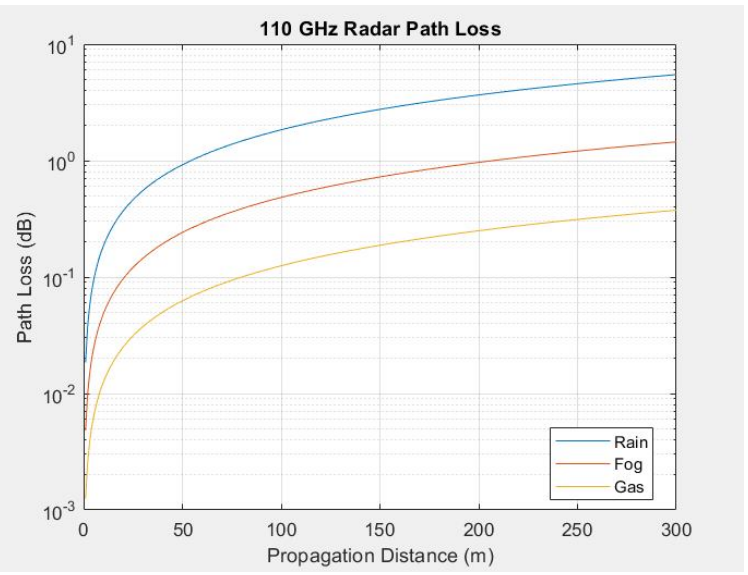

Figure 9: Signal Loss of a 110GHz Radar

$$
\text { L_total }=
$$$$
6.7384
$$

L_analysis $=$

$$
6.7358
$$

Figure 10: Total Loss of a 110GHz Radar

\section{ANALYSIS OF DATA}

In figures 1 to 5 the different attenuations due to very light rain, light rain, moderate rain, heavy rain and extreme rain are graphed respectively. As seen, as the rate of the rain increases from $0.05 \mathrm{~mm} / \mathrm{h}$ to $60 \mathrm{~mm} / \mathrm{h}$ the attenuation also increases with the very light rain having the smallest attenuation and the extreme rain having the biggest attenuation. Both the vertical and horizontal polarizations are considered in the graphing the signal loss due to rain. Although the difference between the vertical and horizontal polarization is small, polarization has an effect on signal loss. It is more obvious on the extreme rain result since the horizontally polarized radar has a greater attenuation compared to the vertically polarized one. The graphs for signal loss due to fog are seen in figures 6 to 7 . For the fog attenuation, the thick fog has a greater signal loss compared to the medium fog. It can also be seen that as the frequency increases, the attenuation also increases. For the atmospheric gas, as seen on the plot in figure 8, there is a strong absorption due to atmospheric gases at around $60 \mathrm{GHz}$. In figure 9, the $110 \mathrm{GHz}$ radar wherein all the previously mentioned environmental stimuli are applied, the loss due to rain is at about $5 \mathrm{dBm}$ at $250 \mathrm{~m}$. Meanwhile, the loss due to fog is at about $1 \mathrm{dBm}$. And lastly, the loss due to atmospheric gas is negligible at less than $0.5 \mathrm{dBm}$. The total power loss of the $110 \mathrm{GHz}$ radar was computed at $6.7385 \mathrm{dBm}$ using the phased array toolbox. In order to verify this, all the losses were added, and the result was $6.7358 \mathrm{dBm}$. Since the two values of the power loss are approximately the same, the computed total loss using the phased array toolbox is correct.

\section{CONCLUSION}

The group was able to simulate the attenuations due to different weather conditions, namely, rain, fog, and atmospheric gas. Based on the results of the different simulations, losses started to appear as soon as the conditions became unfavorable i.e. when light rain was introduced it gradually affects the propagated signal as the weather became more severe from light rain until extreme rain. The fog also contributed to the total attenuation, and similarly, as the fog became thicker, it affected the signal more. Polarization seems to affect the amount of attenuation as well, however, the difference between the loss of a vertically polarized antenna and a horizontally polarized antenna is not noticeable to the point that it is relatively negligible. The effect of atmospheric gas on the propagated signal in the simulation is negligible as well since it is very small. However, this may vary depending on the atmospheric gas present and the amount of pressure applied of the gas. All the mentioned atmospheric factors were simulated on a $110 \mathrm{GHz}$ radar. This helped the researchers understand how environmental conditions affect radio wave propagation. The data that the group has collected provided better insight and understanding as to how the performance of an antenna is affected based solely on the environment it is in. As the weather conditions got worse, so too did the propagation performance of the antenna. The authors recommend that during the design process of an antenna, several contingencies should be put in place so that the attenuations that can have adverse effects can be mitigated. Overall, the group was able to successfully model the effect of various environmental stimuli on radio wave propagation.

\section{RECOMMENDATIONS}

The innovation in wireless communications systems has been constant through recent years as shown by how $5 \mathrm{G}$ is nearing 
maturity, where it will inevitably replace the current $4 \mathrm{G}$ standard. Implementing antenna sites can be quite challenging especially thanks to several sources of attenuation. Since this study merely focused on three different weather conditions, it is possible to model wireless communications systems based on real-world environments such as urban areas or maybe model how future $5 \mathrm{G}$ systems can perform in an especially harsh environment. To be more specific, the group would recommend taking a look into how forests and foliage can add attenuation to signal propagation to get a better picture of how to implement antennas in unserved and underserved rural areas. Furthermore, looking at how more weather conditions can affect antenna performance can be beneficial to areas affected by specific weather conditions such as snowstorms or sandstorms. In addition to that, future research can provide more insight and information about how modeling different antenna configurations can yield different performance. Moreover, modelling antennas to mitigate the performance impact caused by different environments can provide interesting results. Another possible model that can be studied further is the implementation of a radio wave propagation model on irregular terrain. This is to allow for a better understanding as to how radio waves propagate in areas located at or near mountain ranges.

More complex models may also have the capacity to show a 3D render of the radiation pattern of an antenna. This in conjunction with a model of an urban city may assist in better interpretation of the data that the model has produced. Furthermore, the type of antenna could have an impact as to how it propagates radio waves and could perform better than other types of antennas depending on the situation. Additional research on this field can provide useful information to designers, companies, and engineers alike.

\section{REFERENCES}

[1] M. Tinin and S. Knizhin, "On radio wave propagation in multiscale randomly inhomogeneous ionosphere," 2017 Progress in Electromagnetics Research Symposium Spring (PIERS), 2017.

[2] F. Liu, C. Niu, and Y. Yuan, "Radio waves propagate on the clam ocean surface," 2018 33rd Youth Academic Annual Conference of Chinese Association of Automation (YAC), 2018.

[3] J. Fathi and F. Sadikoglu, "Radio Wave Propagation Model for Enhancing Wireless Coverage in Elevator of Buildings," Advances in Intelligent Systems and Computing 10th International Conference on Theory and Application of Soft Computing, Computing with Words and Perceptions - ICSCCW-2019, 463-469, 2019.

[4] I. Elmutasim and I. Mohd, "Examination Rain and Fog Attenuation for Path Loss Prediction in Millimeter Wave Range," Lecture Notes in Electrical Engineering Proceedings of the 11th National Technical Seminar on Unmanned System Technology 2019, 935-946, 2020.

[5] A. E. Pakzad, A. A. Pakzad, and L. Materum, "Proposed joint propagation and reinforcement learning-based television white space ledger," International Journal of
Emerging Trends in Engineering Research, vol. 8, no. 4, pp. 1450-1456, 2020.

[6] E. Masson, P. Combeau, Y. Cocheril, M. Berbineau, L. Aveneau, and R. Vauzelle, "Radio wave propagation in arch-shaped tunnels: Measurements and simulations by asymptotic methods," ComptesRendus Physique, vol. 11, no. 1, pp. 44-53, 2010.

[7] V. Popov, "Cross-polarization effect of radio waves propagation by forest vegetation in wireless communication systems on transport," Procedia Computer Science, vol. 149, pp. 195-201, 2019.

[8] A. Aldhaibani, T. Rahman, and A.Alwarafy, "Radio-propagation measurements and modeling in indoor stairwells at millimeter-wave bands," Physical Communication, vol. 38, 100955, 2020.

[9] O. Simpson and Y. Sun, "LTE RSRP, RSRQ, RSSNR and local topography profile data for RF propagation planning and network optimization in an urban propagation environment," Data in Brief, vol. 21, pp. 1724-1737, 2018.

[10] N. Suzuki and H. Matsuno, "Radio Wave Environment Analysis at Different Locations Based on Frequent Pattern Mining," Procedia Computer Science, vol. 112, pp. 1396-1403, 2017.

[11] A. Africa, "Radio Wave Propagation: Simulation of Free Space Propagation Path Loss," International Journal of Emerging Trends in Engineering Research, vol. 8, no. 2, pp. 281-287, 2020.

[12] G. Feng, J. Huang, and Z. Wu, "Overcoming geometric issues in the multipath propagation of electromagnetic waves using ray tracing and spherical ground surface theory," Optik, vol. 181, pp. 326-337, 2019.

[13]F. Pachón-García, "Modeling ground-wave propagation at MF band in hilly environments through FDTD method and interaction with GIS," AEU - International Journal of Electronics and Communications, vol. 70, no. 8, pp. 981-989, 2016.

[14] I. Kuzichev,I.Vasko, A. Malykhin, and A. Soto-Chavez, "On the ionospheric propagation of VLF waves generated by currents in the lower ionosphere," Journal of Atmospheric and Solar-Terrestrial Physics, vol. 179, pp. 138-148, 2018.

[15]M. Fagre, B. Zossi, J. Chum, E. Yiğit, and A. Elias, "Ionospheric high frequency wave propagation using different IRI hmF2 and foF2 models," Journal of Atmospheric and Solar-Terrestrial Physics, vol. 196, 105141, 2019.

[16]X. Zhang, S. Zhao, R. Song, and D. Zhai, "The propagation features of LF radio waves at topside ionosphere and their variations possibly related to Wenchuan earthquake in 2008," Advances in Space Research, vol. 63, no. 11, pp. 3536-3544, 2019.

[17] K. Saha, K.Parameswaran, and C. Raju, "Tropospheric delay in microwave propagation for tropical atmosphere based on data from the Indian subcontinent," Journal of Atmospheric and Solar-Terrestrial Physics, vol. 69, no. 8, pp. 875-905, 2007.

[18] R. Acharya, "Tropospheric propagation and impairments. Satellite Signal Propagation, Impairments and Mitigation, pp. 159-194, 2017. 
[19]S. Sotiroudis, S.Goudos, and K.Siakavara, "Deep learning for radio propagation: Using image-driven regression to estimate path loss in urban areas," ICT Express, vol. 6, no. 3, pp. 160-165, 2020.

[20]N. Surajudeen-Bakinde, N.Faruk, S. Popoola, M. Salman, A.Oloyede, L. Olawoyin, and C. Calafate, "Path loss predictions for multi-transmitter radio propagation in VHF bands using Adaptive Neuro-Fuzzy Inference System," Engineering Science and Technology, an International Journal, vol. 21, no. 4, pp. 679-691, 2018.

[21] M. Surugiu, I. Petrescu, and R. Gheorghiu, "Analysis of Signal Propagation in Vehicular Communications," Procedia Manufacturing, vol. 46, pp. 754-759, 2020.

[22] N. Hakem, G. Delisle, and Y. Coulibaly, "Radio-wave propagation into an underground mine environment at 2.4 $\mathrm{GHz}, 5.8 \mathrm{GHz}$ and $60 \mathrm{GHz}$," The 8th European Conference on Antennas and Propagation (EuCAP), 2014.

[23] A. Sulyman, A.Alwarafy, G. Maccartney, T. Rappaport, and A. Alsanie, "Directional Radio Propagation Path Loss Models for Millimeter-Wave Wireless Networks in the 28-, 60-, and 73-GHz Bands," IEEE Transactions on Wireless Communications, vol. 15, no. 10, pp. 6939-6947, 2016.

[24] N. Wolff, S. Chevtchenko, A. Wentzel, O. Bengtsson, and W. Heinrich, "Switch-Type Modulators and PAs for Efficient Transmitters in the 5G Wireless Infrastructure," 2018 IEEE MTT-S International Microwave Workshop Series on 5G Hardware and System Technologies (IMWS-5G), 2018.

[25] B. Hannaidh, P. Fitzgerald, H. Berney, R. Lakshmanan, N. Coburn, S. Geary, and B. Mulvey, "Devices and Sensors Applicable to 5G System Implementations," 2018 IEEE MTT-S International Microwave Workshop Series on 5G Hardware and System Technologies (IMWS-5G), 2018.

[26] S. Popoola, A. Atayero, O.Arausi, and V. Matthews, "Path loss dataset for modeling radio wave propagation in smart campus environment," Data in Brief, vol. 17, pp. 1062-1073, 2018.

[27] S.Sotiroudis and K. Siakavara, "Mobile radio propagation path loss prediction using Artificial Neural Networks with optimal input information for urban environments," AEU - International Journal of Electronics and Communications, vol. 69, no. 10, pp. 1453-1463, 2015.

[28] R. Gesner, C. Christodoulou, S. Lane, D. Murrell, E. Hong, and N. Tarasenko, "Modeling the Effects of Gaseous Absorption and Attenuation due to Clouds for a 72 GHz Terrestrial Link," 2019 IEEE International Symposium on Antennas and Propagation and USNC-URSI Radio Science Meeting, 2019.

[29]Z. Zhao, W. Hou, and J. Wang, "Effect of Antenna Location and Polarization on Radio Wave Propagation in Tunnel," 2019 International Conference on Microwave and Millimeter Wave Technology (ICMMT), 2019.

[30] C. Gomes, D. Silva, J. Araújo, H. Gomes, and G. Cavalcante, "Radio-Wave Propagation Model for UHF Band in Different Climatic Conditions with Dyadic Green's Function," Journal of Microwaves,
Optoelectronics and Electromagnetic Applications, vol. 14, no. 1, pp. 60-72, 2015.

[31] K. Igwe, O. Oyedum, M. Ajewole, and A. Aibinu, "Evaluation of some rain attenuation prediction models for satellite communication at Ku and Ka bands," Journal of Atmospheric and Solar-Terrestrial Physics, vol. 188, pp. 52-61, 2019.

[32] A. Africa and J. Lao, "Development of an antenna array radiation model in propagation systems for a low power smart antenna system," International Journal of Emerging Trends in Engineering Research, vol. 8, no. 7, pp. 3373-3378, 2020. 